\title{
A Pretargeted PET Imaging Strategy Based on Bioorthogonal Diels-Alder Click Chemistry
}

\author{
Brian M. Zeglis ${ }^{1}$, Kuntal K. Sevak ${ }^{1}$, Thomas Reiner ${ }^{1}$, Priya Mohindra ${ }^{1}$, Sean D. Carlin ${ }^{1}$, Pat Zanzonico ${ }^{2}$, \\ Ralph Weissleder ${ }^{3}$, and Jason S. Lewis ${ }^{1}$ \\ ${ }^{I}$ Department of Radiology, Memorial Sloan-Kettering Cancer Center, New York, New York; ${ }^{2}$ Department of Medical Physics, Memorial \\ Sloan-Kettering Cancer Center, New York, New York; and ${ }^{3}$ Center for Systems Biology, Massachusetts General Hospital, Boston, Massachusetts
}

The specificity of antibodies have made immunoconjugates promising vectors for the delivery of radioisotopes to cancer cells; however, their long pharmacologic half-lives necessitate the use of radioisotopes with long physical half-lives, a combination that leads to high radiation doses to patients. Therefore, the development of targeting modalities that harness the advantages of antibodies without their pharmacokinetic limitations is desirable. To this end, we report the development of a methodology for pretargeted PET imaging based on the bioorthogonal Diels-Alder click reaction between tetrazine and transcyclooctene. Methods: A proof-ofconcept system based on the A33 antibody, SW1222 colorectal cancer cells, and ${ }^{64} \mathrm{Cu}$ was used. The huA33 antibody was covalently modified with transcyclooctene, and a NOTA-modified tetrazine was synthesized and radiolabeled with ${ }^{64} \mathrm{Cu}$. Pretargeted in vivo biodistribution and PET imaging experiments were performed with athymic nude mice bearing A33 antigen-expressing, SW1222 colorectal cancer xenografts. Results: The huA33 antibody was modified with transcyclooctene to produce a conjugate with high immunoreactivity, and the ${ }^{64} \mathrm{Cu}-\mathrm{NOTA}$-labeled tetrazine ligand was synthesized with greater than $99 \%$ purity and a specific activity of $9-10 \mathrm{MBq} / \mu \mathrm{g}$. For in vivo experiments, mice bearing SW1222 xenografts were injected with transcyclooctene-modified A33; after allowing $24 \mathrm{~h}$ for accumulation of the antibody in the tumor, the mice were injected with ${ }^{64} \mathrm{Cu}$-NOTA-labeled tetrazine for PET imaging and biodistribution experiments. At $12 \mathrm{~h}$ after injection, the retention of uptake in the tumor $(4.1 \pm 0.3$ percent injected dose per gram), coupled with the fecal excretion of excess radioligand, produced images with high tumor-to-background ratios. PET imaging and biodistribution experiments performed using A33 directly labeled with either ${ }^{64} \mathrm{Cu}$ or ${ }^{89} \mathrm{Zr}$ revealed that although absolute tumor uptake was higher with the directly radiolabeled antibodies, the pretargeted system yielded comparable images and tumor-to-muscle ratios at 12 and $24 \mathrm{~h}$ after injection. Further, dosimetry calculations revealed that the ${ }^{64} \mathrm{Cu}$ pretargeting system resulted in only a fraction of the absorbed background dose of $A 33$ directly labeled with ${ }^{89} \mathrm{Zr}$ ( $0.0124 \mathrm{mSv} / \mathrm{MBq}$ vs. $0.4162 \mathrm{mSv} / \mathrm{MBq}$, respectively). Conclusion: The high quality of the images produced by this pretargeting approach, combined with the ability of the methodology to dramatically reduce nontarget radiation doses to patients, marks this system as a strong candidate for clinical translation.

Received Oct. 18, 2012; revision accepted Jan. 28, 2013.

For correspondence or reprints contact: Jason S. Lewis, 1275 York Ave., New York, NY, 10065

E-mail: lewisj2@mskcc.org

Published online May 24, 2013.

COPYRIGHT (C) 2013 by the Society of Nuclear Medicine and Molecular Imaging, Inc.
Key Words: positron emission tomography (PET); pretargeting; click chemistry; antibody; colorectal cancer

J Nucl Med 2013; 54:1389-1396

DOI: 10.2967/jnumed.112.115840

$\mathbf{T}$ he remarkable specificity and affinity of antibodies make them extremely attractive vectors for the delivery of diagnostic and therapeutic radioisotopes to cancer cells (1). Over the last 2 decades, a wide variety of antibody-based radiopharmaceuticals has been developed using isotopes ranging from ${ }^{64} \mathrm{Cu}$ for imaging to ${ }^{225} \mathrm{Ac}$ for therapy. Importantly, the relatively slow pharmacokinetics of antibodies require that the radioactive half-life of the isotope be compatible with the biologic half-life of the target-tissue localization of the vector (2). In practice, this means that antibodies are often labeled with isotopes with multiday physical half-lives. Such long physical half-lives allow time for the antibodies to accumulate in the tumor while simultaneously preventing the premature decay of their radioactive payload. However, this combination of long biologic and physical half-lives gives rise to a critical limiting complication: high activity concentrations in and radiation doses to nontarget organs.

To circumvent this problem, considerable attention has been dedicated to the development of targeting methodologies that combine the advantages of antibodies with the pharmacokinetics of smaller molecules. One particularly appealing method of achieving this balance while still using intact antibodies is termed pretargeting (3-5). Generally, pretargeted methodologies involve 4 steps: the injection into the bloodstream of a bivalent antibody with the ability to bind both an antigen and a radioligand; the slow accumulation of the antibody in the tumor and concomitant clearance of the antibody from the blood; the injection into the bloodstream of the small-molecule radioligand; and the binding of the radioligand to the antibody, followed by the rapid clearance of excess radioactivity. The pharmacokinetics of the small-molecule ligands not only reduces background radiation dose to nontarget organs but also facilitates the use of radioisotopes with short half-lives that would normally be incompatible with antibody-based imaging.

Two strategies for pretargeted systems predominate in the literature. In the first, bispecific antibodies capable of binding both an antigen and a radiolabeled hapten - such as a diethylenetriaminepentaacetic acid-chelated radiometal or a chelator-modified biotin-are used $(6,7)$. In the second strategy, antibodies covalently modified with oligomeric nucleic acids are used as the targeting vectors while radiolabeled, complementary oligonucleotide sequences are 
used as the radioligands (8). Both systems are creative solutions and have proven successful in vivo $(9,10)$. Each, however, possesses significant limitations that threaten their clinical applicability. For the former, these include the complexity and expense associated with the generation of bispecific antibodies and the immunogenicity of streptavidin-based systems; for the latter, both the in vivo stability of the olignucleotides and the expense of using nonnatural nucleic acid analogs are concerns.

In recent years, a chemical methodology has been developed that has the potential to aid in the creation of novel, antibodybased pretargeting systems without these limitations: bioorthogonal click chemistry (11). Although the $[3+2]$ Huisgen cycloaddition between an azide and alkyne remains the preeminent form of click chemistry (12), another promising click ligation variant has recently garnered considerable interest: the rapid, selective, and bioorthogonal inverse electron-demand [4 +2$]$ Diels-Alder cycloaddition between a tetrazine and a strained alkene dienophile (Fig. 1) $(13,14)$. To date, the ligation has been used in a variety of settings, including fluorescence imaging with antibodies and nanoparticles (13) and PET imaging with both ${ }^{18} \mathrm{~F}$ - and radiometal-based probes (15-17). Recently, and most applicable here, 2 reports have been made of pretargeting systems based on the tetrazine-dienophile ligation: one using a small molecule tetrazine moiety equipped with a SPECT isotope and the second using a larger, tetrazine-modified, ${ }^{18} \mathrm{~F}$-labeled dextran-based radioligand $(18,19)$.

Herein, we report the development of a pretargeted PET imaging methodology based on the $[4+2]$ cycloaddition reaction between tetrazine and transcyclooctene (Fig. 2). For proof of concept, we have used a colorectal cancer cell line, the A33 antibody, and the positron-emitting radiometal ${ }^{64} \mathrm{Cu}$ as a model system. Ultimately, we have found that the in vivo click methodology is highly effective at delineating tumor from normal tissue, producing PET images with contrast comparable to that obtained with directly radiolabeled antibodies.

\section{MATERIALS AND METHODS}

\section{Reagents and General Procedures}

All chemicals, unless otherwise noted, were acquired from SigmaAldrich and used as received without further purification. All water used was ultra-pure $\left(>18.2 \mathrm{M} \Omega \mathrm{cm}^{-1}\right)$, and all dimethyl sulfoxide was of molecular biology grade ( $>99.9 \%$ ). Both $2,2^{\prime}, 2^{\prime \prime}$-(2-(4-aminobenzyl)-1,4,7-triazonane-1,4,7-triyl)triacetic acid $\left(\mathrm{NH}_{2}\right.$-Bn-NOTA) and $p$-isothiocyanatobenzyl-desferrioxamine (SCN-DFO) were purchased from Macrocyclics, Inc. Amine-reactive transcyclooctene (TCO-NHS) and amine-reactive tetrazine (Tz-NHS) were synthesized as described previously (13). Humanized A33 antibody was generously provided by the Ludwig Institute for Cancer Immunotherapy.

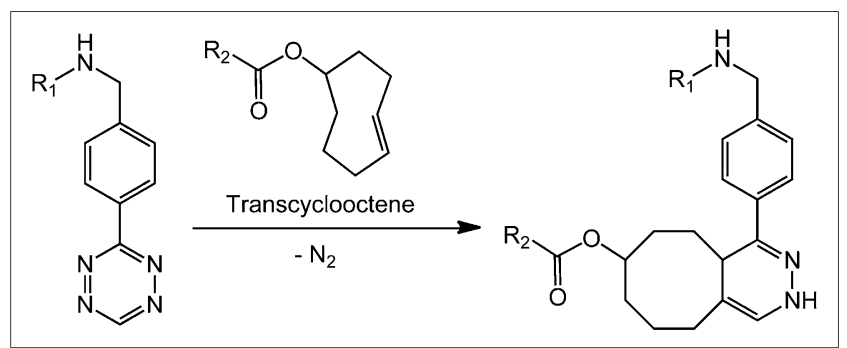

FIGURE 1. Inverse electron-demand Diels-Alder [4 + 2] cycloaddition click ligation between tetrazine and transcyclooctene.
${ }^{64} \mathrm{Cu}$ was purchased from Washington University, St. Louis, where it was produced on the Washington University School of Medicine Cyclotron (model CS-15; Cyclotron Corp.) by the ${ }^{64} \mathrm{Ni}(p, n){ }^{64} \mathrm{Cu}$ reaction and purified to yield ${ }^{64} \mathrm{Cu}-\mathrm{CuCl}_{2}$ with a specific activity of

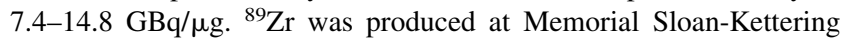
Cancer Center on a TR19/9 cyclotron (Ebco Industries Inc.) via the ${ }^{89} \mathrm{Y}(p, n){ }^{89} \mathrm{Zr}$ reaction and purified to yield ${ }^{89} \mathrm{Zr}$ with a specific activity of $196-496 \mathrm{MBq} / \mu \mathrm{g}$. Activity measurements were made using a CRC-15R Dose Calibrator (Capintec). For the quantification of activities, experimental samples were counted on an Automatic Wizard (2) $\gamma$-Counter (Perkin Elmer). The labeling of antibodies with both ${ }^{64} \mathrm{Cu}$ and ${ }^{89} \mathrm{Zr}$ was monitored using silica gel-impregnated instant thin-layer chromatography (TLC) paper (Pall Corp.) and analyzed on an AR-2000 radio-TLC plate reader (Bioscan Inc.). The human colorectal cancer cell line SW1222 was obtained from the Ludwig Institute for Cancer Immunotherapy and grown by serial passage. All in vivo experiments were performed according to protocols approved by the Memorial SloanKettering Institutional Animal Care and Use Committee

\section{Modification of A33 with TCO-NHS}

A33 $(2 \mathrm{mg})$ was dissolved in $500 \mu \mathrm{L}$ of phosphate-buffered saline (PBS, $\mathrm{pH}$ 7.4), and the $\mathrm{pH}$ of the solution was adjusted to 8.8-9.0 with $\mathrm{NaHCO}_{3}(0.1 \mathrm{M})$. To this solution was added an appropriate volume of TCO-NHS in $N, N$-dimethylformamide $(10 \mathrm{mg} / \mathrm{mL})$ to yield a TCO: mAb reaction stoichiometry of 10:1. The resulting solution was incubated with gentle shaking for $30 \mathrm{~min}$ at room temperature. After $30 \mathrm{~min}$, the modified antibody was purified using centrifugal filter units with a 50,000-Dalton molecular weight cutoff (Amicon Ultra 4; Millipore Corp.) and PBS.

\section{Radiolabeling of Tz-Bn-NOTA with ${ }^{64} \mathrm{Cu}$}

A solution of Tz-Bn-NOTA $(5-6 \mu \mathrm{g})$ in $\mathrm{NH}_{4} \mathrm{OAc}$ buffer $(0.2 \mathrm{M}$, pH 5.5) was prepared. ${ }^{64} \mathrm{CuCl}_{2}$ in $0.1 \mathrm{M} \mathrm{HCl}(74-81 \mathrm{MBq})$ was added to this solution, and the reaction mixture was heated to $80^{\circ} \mathrm{C}$ for $10 \mathrm{~min}$. After heating, the solution was allowed to cool to room temperature and purified via high-performance liquid chromatography (HPLC) (retention time, $9.6 \mathrm{~min}$ ). Solvent was removed via rotary evaporation to yield purified product in an $87 \% \pm 3 \%$ decay-corrected yield with a radiochemical purity of more than $99 \%$ and a specific activity of $8.9 \pm 1.2 \mathrm{MBq} / \mu \mathrm{g}$.

\section{Pretargeted PET Imaging}

Pretargeted PET imaging experiments were conducted on a microPET Focus 120 scanner (Concorde Microsystems). Mice bearing subcutaneous SW1222 xenografts $\left(100-150 \mathrm{~mm}^{3}, 9-12 \mathrm{~d}\right.$ after inoculation) were administered A33-TCO (100 $\mu \mathrm{g}$ in $200 \mu \mathrm{L}$ of $0.9 \%$ sterile saline) via tail vein injection. After $24 \mathrm{~h}$, mice were administered ${ }^{64} \mathrm{Cu}$-Tz-Bn-NOTA $(10.2-12.0 \mathrm{MBq}$ [275-325 $\mu \mathrm{Ci}]$ in 200 $\mu \mathrm{L}$ of $0.9 \%$ sterile saline) via tail vein injection $(t=0)$. Approximately 5 min before PET imaging, mice were anesthetized by inhalation of a $2 \%$ isoflurane (Baxter Healthcare):oxygen gas mixture and placed on the scanner bed; anesthesia was maintained using a $1 \%$ isoflurane:gas mixture. PET data for each mouse were recorded in list mode at various time points between 2 and $18 \mathrm{~h}$ (further technical specifications are given in the supplemental materials, "Methods" section, available online only at http://jnm.snmjournals.org).

\section{RESULTS}

\section{System Design}

The first step in the development of the pretargeting methodology was the design of the model system. Five components needed to be chosen: antibody, tetrazine, dienophile, radionuclide, and chelator. The antibody selected, A33, is a humanized antibody that targets the A33 antigen, a transmembrane glycoprotein 


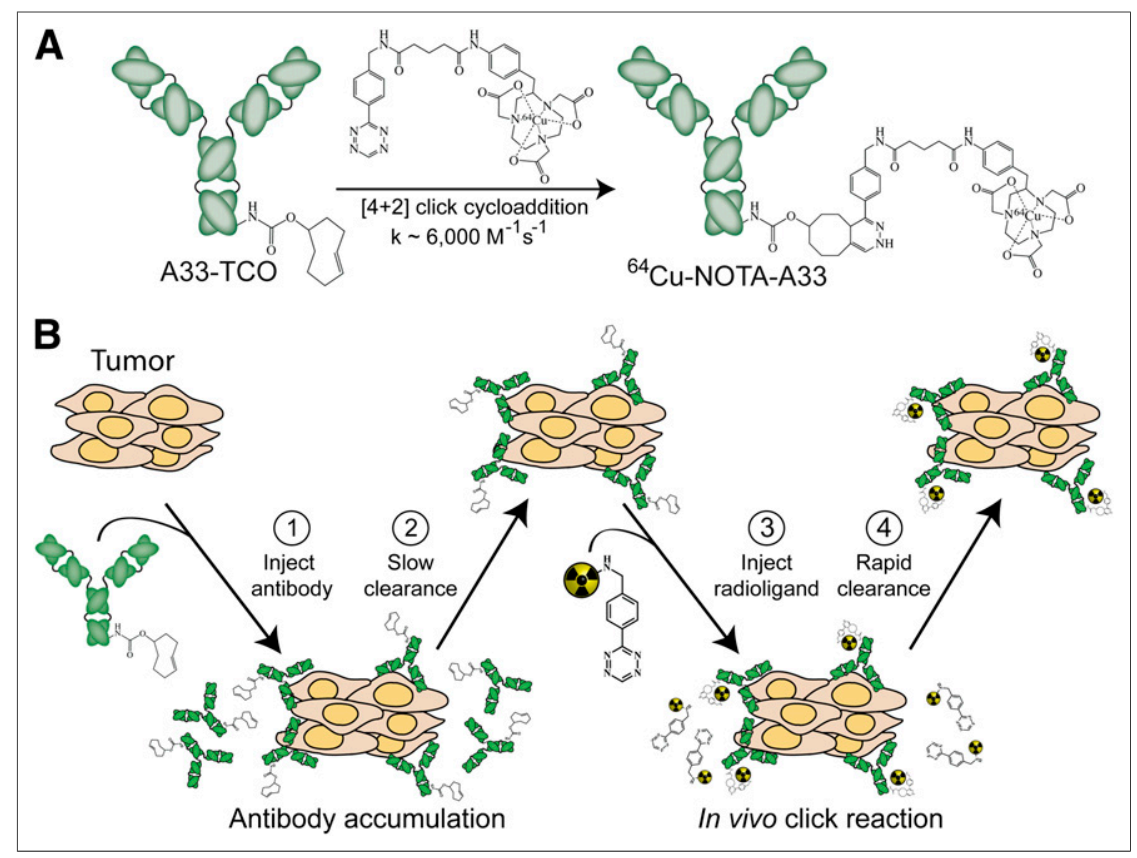

FIGURE 2. Click chemistry-based pretargeting system. (A) Inverse electron-demand Diels-Alder click reaction between A33-TCO and ${ }^{64} \mathrm{Cu}-\mathrm{Tz}-\mathrm{Bn}-\mathrm{NOTA}$. (B) Illustration of the 4 steps of the pretargeting methodology.

present in more than $95 \%$ of human colorectal cancers (20). In addition, because of its association with tight junction proteins, the A33 antigen has been shown to exhibit surface persistence, even when bound to the targeting antibody (21). This is an extremely important trait in the context of pretargeting: the internalization and consequent sequestration of the antibody before the administration of the radioligand would severely reduce the likelihood of in vivo click ligations. 3-(4-benzylamino)-1,2,4,5-tetrazine and transcyclooct4-en-1-yl hydrogen carbonate were chosen as the tetrazine-dienophile click pair because of their convenient conjugation handles and their balance of relative stability with rapid reaction kinetics $\left(k=6,000 \mathrm{M}^{-1} \mathrm{~s}^{-1}\right.$ at $37^{\circ} \mathrm{C}$ ) $(13,14)$. Finally, ${ }^{64} \mathrm{Cu}$ was selected as the radioisotope. Despite the utility and ubiquity of ${ }^{18} \mathrm{~F}$, a radiometal-based system offers greater versatility and modularity. In particular, ${ }^{64} \mathrm{Cu}$ displays favorable imaging characteristics and exhibits a half-life $(12.7 \mathrm{~h})$ normally considered suboptimal for clinical antibody imaging. The choice of ${ }^{64} \mathrm{Cu}$, in turn, narrowed the choice of chelator, and NOTA was selected because of its relatively rapid chelation kinetics and high in vivo stability with ${ }^{64} \mathrm{Cu}(22)$.

\section{Synthesis, Characterization, and Reactivity of Components}

The NOTA-modified tetrazine (Tz-Bn-NOTA) was synthesized in high yield $(>95 \%)$ via peptide coupling from $\mathrm{NH}_{2}$-Bn-NOTA and 3-(4-benzylamino)-1,2,4,5-tetrazine bearing an amine-reactive linker (Fig. 3). The compound was purified using reversed-phase $\mathrm{C}_{18}$ HPLC and characterized by ultraviolet-visible spectroscopy, proton nuclear magnetic resonance spectroscopy, and electrospray ionization mass spectrometry. Tz-Bn-NOTA was, in turn, labeled with ${ }^{64} \mathrm{Cu}$ via incubation with ${ }^{64} \mathrm{Cu}-\mathrm{CuCl}_{2}$ at $90^{\circ} \mathrm{C}$ for $10 \mathrm{~min}$ in $\mathrm{NH}_{4} \mathrm{OAc}$ buffer. The radioligand was obtained in $87 \% \pm 3 \%$ decay-corrected yield and purified via reversed-phase HPLC to more than 98\% radiochemical purity, and its identity was confirmed against a cold $\mathrm{Cu}-\mathrm{Tz}-\mathrm{Bn}$ NOTA standard. The specific activity of the purified ${ }^{64} \mathrm{Cu}-\mathrm{Tz}-\mathrm{Bn}-\mathrm{NOTA}$ was 8.9 $\pm 1.2 \mathrm{MBq} / \mu \mathrm{g}(n=10)$.

The stability of the radioligand was assayed in vitro by incubation in human serum at $37^{\circ} \mathrm{C}$ and subsequent HPLC analysis. The ${ }^{64} \mathrm{Cu}-\mathrm{Tz}-\mathrm{Bn}-\mathrm{NOTA}$ remained relatively stable after $1 \mathrm{~h}$, with $93.0 \% \pm 2.5 \%$ intact. With time, however, decomposition of the ${ }^{64} \mathrm{Cu}-\mathrm{Tz}-\mathrm{Bn}$-NOTA becomes apparent: at $12 \mathrm{~h}$, only $31.6 \% \pm 6.1 \%$ of the radioligand remained intact. In addition, in vivo stability experiments were performed, and these produced similar results, although some acceleration in decomposition can be noted: $77 \% \pm 3 \%$ of the ${ }^{64} \mathrm{Cu}-\mathrm{Tz}-$ Bn-NOTA is intact in the blood after $60 \mathrm{~min}$, followed by $47 \% \pm$ $2 \%$ after $2 \mathrm{~h}$ and $22 \% \pm 3 \%$ after $6 \mathrm{~h}$. Although greater stability may be preferable, the speed of the cycloaddition reaction and the rapid pharmacokinetics of the radioligand led us to believe it unlikely that poor serum stability at later time points would limit the system (Supplemental Figs. 1 and 2; Supplemental Table 1).

The TCO-modified A33 (A33-TCO) was constructed via reaction of the antibody with 10 molar equivalents of the $\mathrm{N}$-hydrosuccinimidyl ester of TCO (TCO-NHS) for $30 \mathrm{~min}$ at room temperature in PBS adjusted to $\mathrm{pH} 8.8-9.0$ with $\mathrm{NaHCO}_{3}(0.1 \mathrm{M})$, and the bioconjugate was purified using centrifugal filtration. To determine the number of TCO per monoclonal antibody, the antibody was ligated with a 50-fold molar excess of Tz-Bn-NOTA in PBS (pH 7.4) and purified via size-exclusion chromatography. An isotopic dilution was performed on this NOTA-modified product to determine a degree of labeling of $5.0 \pm 0.9 \mathrm{NOTA} / \mathrm{mAb}$, in turn suggesting a degree of labeling of $5 \mathrm{TCO} / \mathrm{mAb}$, given the quantitative nature of the click ligation (18).

With the 2 system components in hand, the reaction between A33-TCO and ${ }^{64} \mathrm{Cu}-\mathrm{Tz}-\mathrm{Bn}-\mathrm{NOTA}$ was next investigated. A33TCO was incubated with ${ }^{64} \mathrm{Cu}-\mathrm{Tz}-\mathrm{Bn}$-NOTA in $1: 5,1: 1$, or $5: 1$ Tz-to-mAb molar ratios in either PBS or serum at $37^{\circ} \mathrm{C}$ for $30 \mathrm{~min}$. As analyzed by radio-TLC, the reaction proceeded to

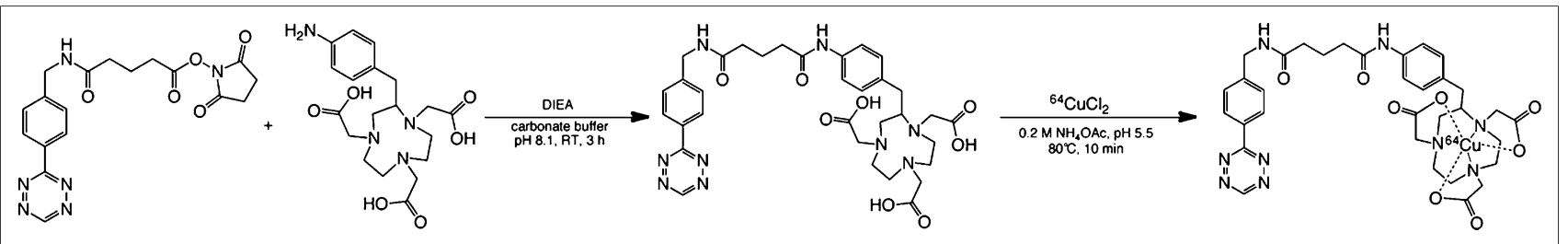

FIGURE 3. Synthesis of ${ }^{64} \mathrm{Cu}-\mathrm{Tz}-\mathrm{Bn}-\mathrm{NOTA}$. 
near-complete conversion, with the $1: 5 \mathrm{Tz}$-to-mAb reaction providing yields of $91.2 \% \pm 0.9 \%$ and $89.6 \% \pm 1.8 \%$ in PBS and serum, respectively, with only slightly diminished yields with the other ratios (18). As negative controls, the reactions of free ${ }^{64} \mathrm{Cu}$ with A33-TCO and ${ }^{64} \mathrm{Cu}-\mathrm{Tz}-\mathrm{Bn}$-NOTA with unmodified A33 were investigated, and in each case less than $1 \%$ association of the activity with the antibody was observed (Supplemental Tables 2 and 3).

Finally, to probe the effect of the TCO conjugation on the immunoreactivity of the antibody, A33-TCO was reacted with a 5fold excess of ${ }^{64} \mathrm{Cu}-\mathrm{Tz}-\mathrm{Bn}-\mathrm{NOTA}$ in PBS and purified via sizeexclusion chromatography to form ${ }^{64} \mathrm{Cu}-\mathrm{NOTA}-\mathrm{A} 33$ in more than $99 \%$ radiochemical purity and a specific activity of $125.8 \pm 11.1$ $\mathrm{MBq} / \mathrm{mg}$ (Supplemental Figs. 3-5). Immunoreactivity assays with A33 antigen-expressing SW1222 human colorectal cancer cells yielded an immunoreactivity for the bioconjugate of $95.6 \% \pm 4.3 \%$.

\section{Pretargeted PET Imaging and Biodistribution}

After the synthesis and characterization of the components of the system, the next step was to perform in vivo pretargeted biodistribution and PET imaging experiments. Not surprisingly, one of the most important variables in the development of a pretargeted system is the length of the interval between the injection of the antibody and the administration of the radioligand. In this case, biodistribution studies with clicked and preassembled ${ }^{64} \mathrm{Cu}-$ NOTA-A33 (see the "Discussion" section) indicate that the maximum loading of ${ }^{64} \mathrm{Cu}-\mathrm{NOTA}-\mathrm{A} 33$, and thus by proxy A33-TCO, in the tumor occurs at $24 \mathrm{~h}$ after injection, and relatively little antibody remains in the blood pool at the same time point. Thus, it becomes clear that $24-48 \mathrm{~h}$ represents a promising interval between the administration of antibody and the subsequent injection of radioligand.

For the pretargeted biodistribution experiments, mice bearing subcutaneous SW1222 xenografts were administered A33-TCO $(100 \mu \mathrm{g})$ via tail vein injection. After a 24-h interval for the accumulation of the antibody in the tumor and its simultaneous clearance from the blood, the mice were administered ${ }^{64} \mathrm{Cu}-\mathrm{Tz}$ Bn-NOTA (0.55-0.75 MBq, 0.06-0.09 $\mu \mathrm{g}$, a 0.14-0.18 Tz-to-A33 ratio) via tail vein injection. The data reveal rapid accumulation and retention of radioactivity in the tumor, with $4.1 \pm 0.3$ percent injected dose per gram $(\% \mathrm{ID} / \mathrm{g})$ at $1 \mathrm{~h}$ after injection, $4.2 \pm 0.8$ $\% \mathrm{ID} / \mathrm{g}$ at $12 \mathrm{~h}$, and $4.0 \pm 0.9 \% \mathrm{ID} / \mathrm{g}$ at $24 \mathrm{~h}$ (Table 1; Supplemental Tables 4 and 5). The compound is excreted through the feces, with high levels of activity at $1 \mathrm{~h}$ after injection dropping to $2.5 \pm 0.1 \% \mathrm{ID} / \mathrm{g}$ at $12 \mathrm{~h}$. The amount of uptake in all other tissues remains low, generally less than $1 \% \mathrm{ID} / \mathrm{g}$. The rapid and steady uptake in the tumor indicates that ${ }^{64} \mathrm{Cu}-\mathrm{Tz}-\mathrm{Bn}$-NOTA is clicking with A33-TCO localized at the tumor, rather than clicking with A33-TCO in the blood pool that subsequently accumulates in the tumor.

PET imaging experiments were performed in a similar manner; the only change was the higher amount of injected activity (10.212.0 MBq, 1.2-1.4 $\mu \mathrm{g}$, a 2.5-2.8 Tz-to-A33 ratio) (Fig. 4; Supplemental Figs. 6 and 7). Tumor uptake is apparent at $2 \mathrm{~h}$ after injection, although consistent with the biodistribution, it is much less than the uptake in the gut (feces). However, over the course of the experiment, the fecal radioactivity clears dramatically, resulting in excellent delineation of tumor from background by $12 \mathrm{~h}$ after injection, in agreement with the $26.6 \pm 6.6$ tumor-to-muscle activity concentration ratio measured at the same time point in the biodistribution study.

Imaging experiments in which $300 \mu \mathrm{g}$ of A33-TCO were administered showed similar results; however, these experiments revealed higher activity levels in the blood, suggesting a greater
TABLE 1

Biodistribution Data for In Vivo ${ }^{64} \mathrm{Cu}$ Pretargeting Experiment

\begin{tabular}{lrccc}
\hline \multicolumn{1}{c}{ Organ } & \multicolumn{1}{c}{$1 \mathrm{~h}$} & $4 \mathrm{~h}$ & $12 \mathrm{~h}$ & $24 \mathrm{~h}$ \\
\hline Blood & $3.5 \pm 0.6$ & $2.6 \pm 0.8$ & $2.3 \pm 0.4$ & $2.1 \pm 0.5$ \\
\hline Tumor & $4.1 \pm 0.3$ & $4.1 \pm 0.6$ & $4.2 \pm 0.8$ & $4.0 \pm 0.9$ \\
Heart & $1.1 \pm 0.2$ & $0.9 \pm 0.3$ & $0.9 \pm 0.1$ & $0.8 \pm 0.2$ \\
Lung & $1.6 \pm 0.5$ & $1.6 \pm 0.4$ & $1.1 \pm 0.4$ & $1.0 \pm 0.3$ \\
Liver & $2.2 \pm 0.3$ & $1.3 \pm 0.3$ & $0.9 \pm 0.2$ & $1.1 \pm 0.2$ \\
Spleen & $0.6 \pm 0.1$ & $0.5 \pm 0.2$ & $0.6 \pm 0.3$ & $0.4 \pm 0.1$ \\
Stomach & $0.5 \pm 0.1$ & $0.3 \pm 0.1$ & $0.5 \pm 0.6$ & $0.2 \pm 0.0$ \\
Large intestine & $0.5 \pm 0.0$ & $3.1 \pm 1.1$ & $2.3 \pm 1.0$ & $1.1 \pm 0.4$ \\
Feces & $11.9 \pm 4.4$ & $8.8 \pm 3.4$ & $2.5 \pm 0.1$ & $1.4 \pm 0.5$ \\
Small intestine & $0.0 \pm 0.0$ & $0.4 \pm 0.1$ & $0.8 \pm 0.5$ & $0.3 \pm 0.0$ \\
Kidney & $1.3 \pm 0.1$ & $0.9 \pm 0.3$ & $0.9 \pm 0.3$ & $0.7 \pm 0.2$ \\
Muscle & $0.2 \pm 0.0$ & $0.1 \pm 0.0$ & $0.2 \pm 0.0$ & $0.1 \pm 0.0$ \\
Bone & $0.3 \pm 0.2$ & $0.3 \pm 0.2$ & $0.4 \pm 0.1$ & $0.3 \pm 0.1$ \\
Skin & $0.3 \pm 0.1$ & $0.3 \pm 0.1$ & $0.4 \pm 0.1$ & $0.5 \pm 0.2$
\end{tabular}

Values are $\% \mathrm{ID} / \mathrm{g} \pm \mathrm{SD}$. Mice $(n=4)$ bearing subcutaneous SW1222 xenografts were administered A33-TCO $(100 \mu \mathrm{g})$ via tail vein injection. After $24 \mathrm{~h}$, the same mice were administered ${ }^{64} \mathrm{Cu}-\mathrm{Tz}-$ Bn-NOTA (0.55-0.75 MBq [15-20 $\mu \mathrm{Ci}])$ via tail vein injection $(t=0)$.

incidence of in vivo click reactions in the blood in addition to ligations at the tumor (Supplemental Figs. 8 and 9; Supplemental Tables 6 and 7). Similarly, imaging experiments performed with a pretargeting interval of $12 \mathrm{~h}$ resulted in a higher degree of activity in the blood, likely for the same reason. Control imaging experiments using ${ }^{64} \mathrm{Cu}-\mathrm{Tz}-\mathrm{Bn}$-NOTA alone, unmodified A33 rather than A33-TCO, and a vast excess of unlabeled tetrazine resulted in minimal uptake of activity in the tumor, in all cases less than $0.25 \% \mathrm{ID} / \mathrm{g}$. Further, pretargeting with a nonspecific IgGTCO construct resulted in apparent yet slight tumor uptake $(<1.5$ $\% \mathrm{ID} / \mathrm{g}$ ), not surprising given the tendency of nontargeted antibodies to accumulate in tumors because of the enhanced permeability and retention effect (Supplemental Figs. 10-14) (23). Finally, although preliminary pilot imaging experiments with longer pretargeting intervals (e.g., $72 \mathrm{~h}$ ) strongly suggest that the 24-h interval used strikes the optimal balance between tumor uptake and background clearance, further interval optimization studies are currently under way in our laboratory.

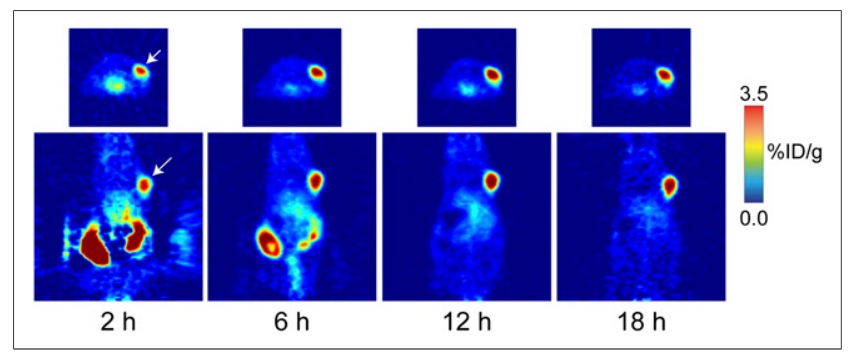

FIGURE 4. PET images of ${ }^{64} \mathrm{Cu}-\mathrm{Tz}-\mathrm{Bn}-\mathrm{NOTA} / \mathrm{A} 33-\mathrm{TCO}$ pretargeting strategy. Mice bearing subcutaneous SW1222 xenografts (100$150 \mathrm{~mm}^{3}$, arrow) were administered A33-TCO $(100 \mu \mathrm{g})$ via tail vein injection. After $24 \mathrm{~h}$, the same mice were administered ${ }^{64} \mathrm{Cu}-\mathrm{Tz}-\mathrm{Bn}$ NOTA (10.2-12.0 MBq [275-325 $\mu \mathrm{Ci}$ ], 1.2-1.4 $\mu \mathrm{g}$, for 2.5-2.8 Tzto-A33 ratio) via tail vein injection. Transverse (top) and coronal (bottom) planar images intersect the center of the tumors. 
Multimodality Imaging, Autoradiography, and

Fluorescence Microscopy

Although the PET imaging experiments form the core of this investigation, they only allow us to monitor the fate of 1 of the 2 components in the system: the radioligand. To circumvent this issue, A33-TCO was modified with the near-infrared fluorophore AlexaFluor680 (AF680) to yield A33-TCO-AF680 with a degree of labeling of $1.6 \mathrm{AF} 680 / \mathrm{mAb}$. Tumor-bearing mice were then injected with A33-TCO-AF680 (100 $\mu \mathrm{g})$, imaged with fluorescence at $24 \mathrm{~h}$ after antibody injection, injected immediately thereafter with ${ }^{64} \mathrm{Cu}$-Tz-Bn-NOTA (10.2-12.0 MBq [275-325 $\left.\left.\mu \mathrm{Ci}\right]\right)$, and then imaged with both fluorescence and PET $12 \mathrm{~h}$ after radiotracer injection. Not surprisingly, the PET imaging results from these experiments clearly mirror those reported above for the nonfluorescent antibody (Supplemental Figs. 15-17).

More important than the in vivo imaging experiments, this multimodality approach facilitated the tracking of the 2 system components at the microscopic level via autoradiography and fluorescence microscopy (Fig. 5). To this end, ex vivo analysis of resected tumors was performed to determine the relative distributions of A33-TCO-AF680 and ${ }^{64} \mathrm{Cu}-\mathrm{Tz}-\mathrm{Bn}-\mathrm{NOTA}$. A close correspondence of the 2 components was observed, with regions of high A33-TCO-AF680 uptake spatially matching regions of high ${ }^{64} \mathrm{Cu}$-Tz-Bn-NOTA uptake. Further, A33-TCO-AF680 was associated exclusively with regions containing tumor cells and did not appear to associate with stromal or muscle tissue. More than any other piece of data in this investigation, the clear and striking microscopic colocalization of the fluorescently labeled A33-TCO and the ${ }^{64} \mathrm{Cu}-\mathrm{Tz}-\mathrm{Bn}-\mathrm{NOTA}$ illustrates the occurrence of the in vivo click ligation. Finally, A33-TCO-AF680 shows a distinct cell surface distribution, which is easily visualized when compared with the nuclear counterstain 4',6-diamidino-2-phenylindole and is consistent with the cell surface expression of the A33 antigen.

\section{DISCUSSION}

Ultimately, an in vivo comparison with the preassembled and purified ${ }^{64} \mathrm{Cu}$-NOTA-A33 construct is essential for the evaluation of the efficacy of this pretargeted system. To this end, the ${ }^{64} \mathrm{Cu}-$ labeled antibody was synthesized as described above and purified to more than $99 \%$ radiochemical purity, with a specific activity of $125.8 \pm 11.1 \mathrm{MBq} / \mathrm{mg}$ and an immunoreactivity of more than $95 \%$. In the biodistribution experiment, nude mice bearing SW1222 xenografts in the right shoulder were injected with ${ }^{64} \mathrm{Cu}-\mathrm{NOTA}-\mathrm{A} 33(0.55-0.75 \mathrm{MBq}, 4-6 \mu \mathrm{g})$ and were euthanized at $4,12,24$, and $48 \mathrm{~h}$, followed by the collection and weighing of tissues and assay of ${ }^{64} \mathrm{Cu}$ activity in each tissue.

High specific uptake of the radiotracer was observed in the SW1222 xenografts, with the \%ID/g increasing from $18.2 \pm 3.0$ at $4 \mathrm{~h}$ to $35.0 \pm 3.8$ at $48 \mathrm{~h}$. As is typical of antibody-based imaging, a concomitant decrease in the $\% \mathrm{ID} / \mathrm{g}$ in the blood also occurred over the course of the experiment. The organs with the highest background uptake were the lungs, liver, and spleen, though the uptake in these organs was at its highest point at $4 \mathrm{~h}$; by $48 \mathrm{~h}$, the tumor-to-organ ratios for each of these organs were $20.8 \pm 13.1$, $10.0 \pm 4.5$, and $19.8 \pm 5.4$, respectively (Supplemental Tables 8-11).

These biodistribution data were corroborated by small-animal PET imaging. In these experiments, tumor-bearing mice were injected with ${ }^{64} \mathrm{Cu}-\mathrm{NOTA}-\mathrm{A} 33$, and PET images were acquired at various time points between 4 and $48 \mathrm{~h}$. The results clearly indicate that the ${ }^{64} \mathrm{Cu}$-NOTA-A33 constructs are taken up in the

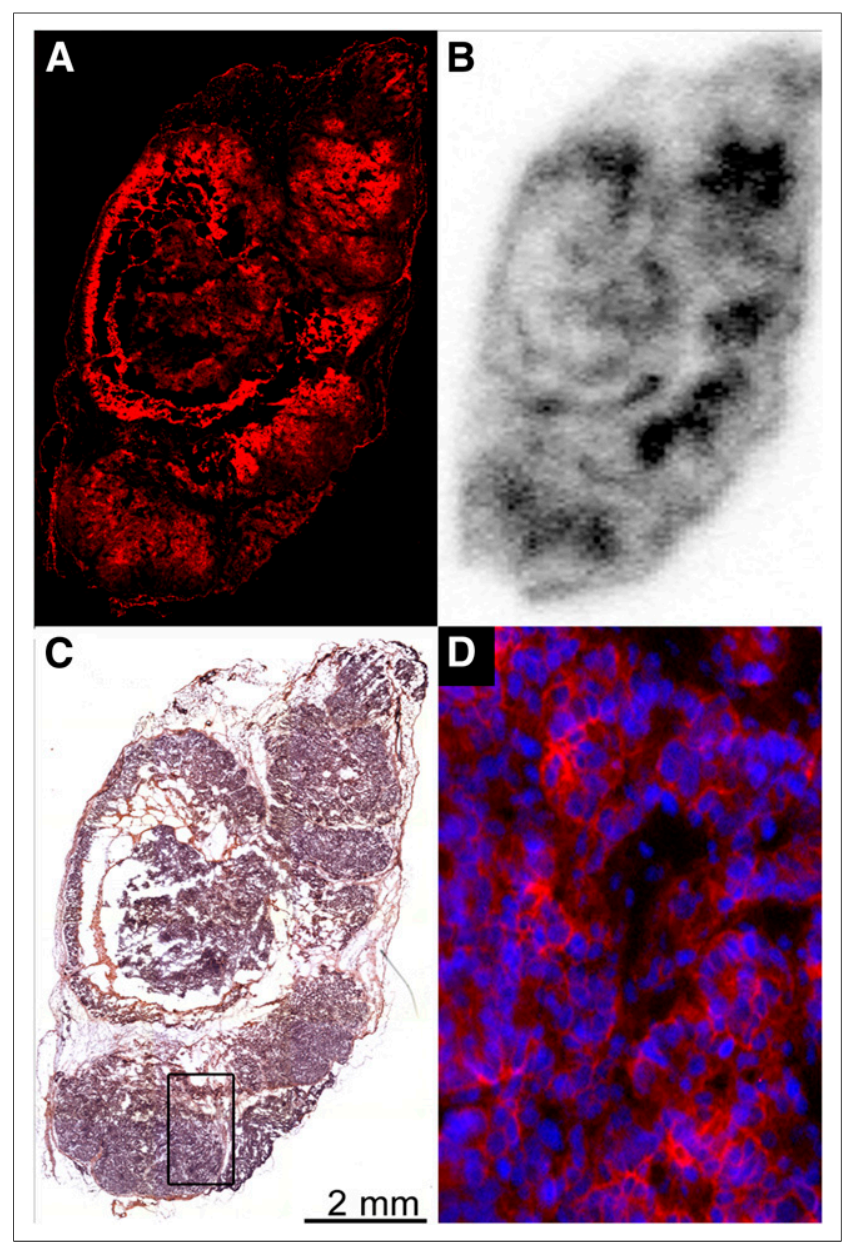

FIGURE 5. Autoradiography, histology, and fluorescence microscopy of resected SW1222 xenografts from multimodality pretargeting experiment: fluorescence microscopy indicating A33-TCO-AF680 localization (red) (A), autoradiography indicating ${ }^{64} \mathrm{Cu}-\mathrm{Tz}-\mathrm{Bn}-\mathrm{NOTA}$ localization $(B)$, hematoxylin and eosin staining $(C)$, and fluorescence microscopy images with A33-TCO-AF680 (red) and 4',6-diamidino-2phenylindolel nuclear counterstain (blue) corresponding to box in C (D).

antigen-expressing SW1222 tumor (Fig. 6; Supplemental Fig. 18). High blood-pool activity and background uptake are evident at early time points, but over the course of the experiment, the tumor signal increases significantly to a point at which it is by far the most prominent feature in the image.

As expected for an intact antibody, the ${ }^{64} \mathrm{Cu}-\mathrm{NOTA}-\mathrm{A} 33$ displays higher absolute uptake in the tumor than the pretargeted system: $33.1 \pm 7.0 \% \mathrm{ID} / \mathrm{g}$ at $24 \mathrm{~h}$, compared with $4.0 \pm 0.9$ $\% \mathrm{ID} / \mathrm{g}$ for the click chemistry approach at the same time point. However, for imaging purposes, the far more important question is target-to-background ratio, and indeed, when speaking in terms of relative uptake, the methodologies become far more similar. At 24 $\mathrm{h}$ after injection, the pretargeting methodology provides a tumorto-muscle activity ratio that is statistically identical to that of ${ }^{64} \mathrm{Cu}$-NOTA-A33: $27.0 \pm 7.4$ versus $33.8 \pm 6.7$, respectively. In addition, at all time points assayed, the uptake in nontarget tissues is significantly higher for the intact antibody than for the pretargeting system in all organs save the large intestine.

Not surprisingly given the pharmacokinetics of intact antibodies, at later time points, the tumor-to-muscle activity ratio for 
the intact ${ }^{64} \mathrm{Cu}-\mathrm{NOTA}-\mathrm{A} 33$ surpasses the maximum values measured for the pretargeting system, reaching $52.2 \pm 14.7$ at $48 \mathrm{~h}$. Likewise, whereas the tumor-to-blood ratios of ${ }^{64} \mathrm{Cu}-\mathrm{NOTA}-\mathrm{A} 33$ and the pretargeting system are comparable at $24 \mathrm{~h}(2.9 \pm 0.4$ and $1.9 \pm 0.6$, respectively), at $48 \mathrm{~h}$, the tumor-to-blood ratio of ${ }^{64} \mathrm{Cu}-$ NOTA-A33 (24.5 \pm 11.6$)$ exceeds the maximum ratio observed with the pretargeting system. Yet despite the high absolute and relative uptake of ${ }^{64} \mathrm{Cu}-\mathrm{NOTA}-\mathrm{A} 33$ at these later time points, the data clearly illustrate that the pretargeted system represents a qualitatively and quantitatively comparable alternative to ${ }^{64} \mathrm{Cu}$-based antibody imaging.

The preceding comparison to ${ }^{64} \mathrm{Cu}-\mathrm{NOTA}-\mathrm{A} 33$ undoubtedly provides the most direct measure of the comparative efficacy of the pretargeting system. However, the recent and rapid advent of clinical ${ }^{89} \mathrm{Zr}$-immunoPET likely means that many of the antibodybased imaging agents in the future will be labeled with ${ }^{89} \mathrm{Zr}$. Thus, we believe that a comparison to directly labeled ${ }^{89} \mathrm{Zr}$-DFO-A33 could also be valuable by providing insight into the merits of ${ }^{64} \mathrm{Cu}$-based pretargeting as an alternative to antibodies labeled with ${ }^{89} \mathrm{Zr}$. To this end, ${ }^{89} \mathrm{Zr}$-DFO-A33 was synthesized by modifying A33 with the ${ }^{89} \mathrm{Zr}$ chelator desferrioxamine via isothiocyanate coupling $(3.5 \pm 1.1 \mathrm{DFO} / \mathrm{A} 33)$ and radiolabeling this construct with ${ }^{89} \mathrm{Zr}$ using standard procedures (2). The purified radiopharmaceutical exhibited more than $99 \%$ radiochemical purity, an immunoreactivity of $92 \% \pm 5 \%$, more than $95 \%$ stability over $7 \mathrm{~d}$ at $37^{\circ} \mathrm{C}$, and a specific activity of $159.1 \pm 22.2 \mathrm{MBq} / \mathrm{mg}$ (Supplemental Figs. 19-21).

For both PET imaging and biodistribution experiments, SW1222 tumor-bearing animals were injected with ${ }^{89} \mathrm{Zr}$-DFOA33 (10.2-12.0 MBq for PET, 0.55-0.75 MBq for biodistribution) and subsequently imaged or euthanized for biodistribution at time points between 4 and $120 \mathrm{~h}$ after injection. Both the PET and the biodistribution experiments display all the hallmarks of ${ }^{89} \mathrm{Zr}$-based antibody imaging (Fig. 7). High early levels of activity in the blood $(58.4 \pm 8.5 \% \mathrm{ID} / \mathrm{g}$ at $4 \mathrm{~h})$ decrease over the first $48 \mathrm{~h}$ as the activity levels in the tumor increase dramatically to a maximum of $44.7 \pm 10.5 \% \mathrm{ID} / \mathrm{g}$ at $48 \mathrm{~h}$. All other tissues exhibit much lower activities after $48 \mathrm{~h}$ with the exception of bone, which displays uptake values of approximately $10 \% \mathrm{ID} / \mathrm{g}$, a consequence of the mineralization of liberated ${ }^{89} \mathrm{Zr}^{4+}$ in bone (Supplemental Fig. 22; Supplemental Tables 12 and 13).

In comparative terms, the directly labeled ${ }^{89} \mathrm{Zr}$-DFO-A33, like ${ }^{64} \mathrm{Cu}-\mathrm{NOTA}-\mathrm{A} 33$, is characterized by higher absolute tumor uptake, with $43.3 \pm 9.0 \% \mathrm{ID} / \mathrm{g}$ in the xenograft at $24 \mathrm{~h}$ compared with $4.0 \pm 0.9 \% \mathrm{ID} / \mathrm{g}$ for the pretargeting system. Again, however, the pretargeting methodology excels with regard to the more important metric: tumor-to-background ratio. Here, the pretargeting approach produces a higher tumor-to-muscle activity ratio than ${ }^{89} \mathrm{Zr}$-DFO-A33 at $24 \mathrm{~h}$ after injection $(27.0 \pm 7.4$ vs. $12.7 \pm$ 3.3 , respectively) as well as a comparable tumor-to-blood activity ratio at the same time point $(1.9 \pm 0.6$ vs. $2.4 \pm 0.7$, respectively). Further, the background associated with the intact antibody is significantly higher than in the pretargeted system.

Not surprisingly, though, both the absolute uptake and the tumor-to-background ratios for ${ }^{89} \mathrm{Zr}$-DFO-A33 increase further at later time points. For example, despite the higher tumor-to-muscle ratio produced by the pretargeting approach at $24 \mathrm{~h}$, the tumor-tomuscle activity ratios for ${ }^{89} \mathrm{Zr}$-DFO-A33 surpass this value at later time points, ultimately reaching a maximum of $57.7 \pm 7.0$ at $72 \mathrm{~h}$.

Critically, for both ${ }^{64} \mathrm{Cu}-\mathrm{NOTA}-\mathrm{A} 33$ and ${ }^{89} \mathrm{Zr}-\mathrm{DFO}-\mathrm{A} 33$, the high absolute uptake values and tumor-to-background ratios ob-

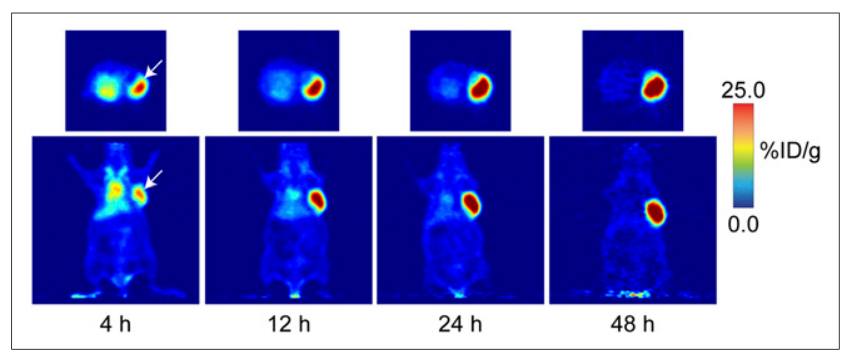

FIGURE 6. PET imaging with ${ }^{64} \mathrm{Cu}-\mathrm{NOTA}-\mathrm{A} 33$. Mice bearing subcutaneous SW1222 xenografts (100-150 mm ${ }^{3}$, arrow) were administered ${ }^{64} \mathrm{Cu}-\mathrm{NOTA}-\mathrm{A} 33(10.2-12.0 \mathrm{MBq}$ [275-325 $\mu \mathrm{Ci}]$ ) via tail vein injection and imaged between 4 and $48 \mathrm{~h}$ after injection. Transverse and coronal planar images intersect the center of the tumors.

served at later time points do not come without cost. In both cases $-{ }^{89} \mathrm{Zr}$, in particular - the pharmacokinetics of the antibodies mean that high levels of radioactivity persist in the patient for extended periods of time; although these levels of activity are often highest in the tumor, the levels in background organs are relatively high as well. It is in this regard that the pretargeted strategy offers an exciting and innovative alternative, for the data clearly indicate that the pretargeted approach produces images comparable to those created with directly radiolabeled antibodies but with both accelerated pharmacokinetics and dramatically lower background uptake in nontarget tissues.

This discussion leads us to another potential advantage to consider for the pretargeting system: reduced radiation dose to nontarget tissues. Using biodistribution data, we performed dosimetry calculations for the pretargeting system, ${ }^{64} \mathrm{Cu}-\mathrm{NOTA}-\mathrm{A} 33$, and ${ }^{89} \mathrm{Zr}$-DFO-A33 using the OLINDA computer program to determine mean organ absorbed doses and effective dose in $\mathrm{mGy} / \mathrm{MBq}$ and $\mathrm{mSv} / \mathrm{MBq}$ respectively (Table 2) (24). The data indicate that the pretargeting system provides a significant dosimetric advantage, compared with the ${ }^{89} \mathrm{Zr}$-labeled antibody: the effective dose with ${ }^{64} \mathrm{Cu}$ pretargeting is $0.0124 \mathrm{mSv} / \mathrm{MBq}$ whereas that due to ${ }^{89} \mathrm{Zr}$ DFO-A33 is $0.416 \mathrm{mSv} / \mathrm{MBq}$. A more pronounced disparity between the two exists when considering the mean absorbed dose to the bone, specifically red marrow and osteogenic cells; with ${ }^{89} \mathrm{Zr}$ DFO-A33, the dose to these tissues is 0.843 and $1.646 \mathrm{mGy} / \mathrm{MBq}$, respectively, compared with 0.0413 and 0.0230 with ${ }^{64} \mathrm{Cu}$ pretargeting. Not surprisingly given the shorter half-life of ${ }^{64} \mathrm{Cu}$, these advantages are not as pronounced when comparing the pretargeting system to ${ }^{64} \mathrm{Cu}-\mathrm{NOTA}-\mathrm{A} 33$. The effective dose of ${ }^{64} \mathrm{Cu}-\mathrm{NOTA}-\mathrm{A} 33$ is 0.0359 $\mathrm{mSv} / \mathrm{MBq}$, approximately 3 times larger than the pretargeting value of $0.0124 \mathrm{mSv} / \mathrm{MBq}$. Thus, whereas the dosimetric differences are somewhat attenuated when making comparisons to the

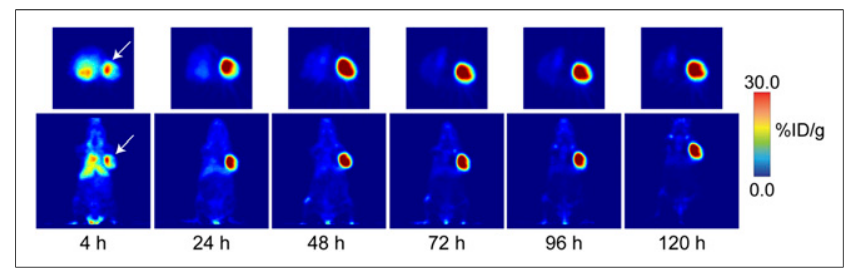

FIGURE 7. PET imaging with ${ }^{89} \mathrm{Zr}$-DFO-A33. Mice bearing subcutaneous SW1222 xenografts $\left(100-150 \mathrm{~mm}^{3}\right.$, arrow) were administered ${ }^{89} \mathrm{Zr}$-DFO-A33 (10.2-12.0 MBq [275-325 $\left.\mu \mathrm{Ci}\right]$ ) via tail vein injection and imaged between 4 and $120 \mathrm{~h}$ after injection. Transverse and coronal planar images intersect the center of the tumors. 
${ }^{64} \mathrm{Cu}$-labeled antibody, it becomes clear that the pretargeting system provides qualitatively and quantitatively comparable imaging results at only a fraction of the background radiation dose of the directly labeled antibodies, particularly the ${ }^{89} \mathrm{Zr}$-labeled construct.

\section{CONCLUSION}

Herein, we have reported the development of a modular pretargeting system for PET imaging based on the inverse electron-demand Diels-Alder cycloaddition between tetrazine and transcyclooctene. In a model system, we have found that this in vivo click methodology produces high-quality images with tumor-to-background contrast comparable to that achieved with directly radiolabeled antibodies. Further, the system facilitates the production of these images at a dramatically reduced absorbed dose to nontarget organs compared with imaging with a ${ }^{89} \mathrm{Zr}$-labeled antibody.

Despite these successes, the system is not without slight limitations. First, the system requires that the antibody either be noninternalizing or have a slow rate of internalization; although this applies to both A33 and several other clinically interesting antibodies (e.g., CC49), there are certainly other clinically relevant antibodies that are known to internalize upon antigen binding and thus would be much more challenging to use in a pretargeted strategy. And second, the relatively slow fecal excretion of the excess radioligand limits both the ability to image abdominal tumors rapidly after the administration of the radio- ligand and the clinical use of radionuclides with short half-lives, such as ${ }^{18} \mathrm{~F}$ or ${ }^{68} \mathrm{Ga}$. Currently, experiments are under way to develop novel tetrazine constructs that have altered pharmacokinetics and pharmacodynamics and thus should enable more rapid imaging. However, these changes may not be strictly necessary in the clinic. If other methods for clearing radioactive feces from the patient are adopted, images can be recorded at earlier time points, and radionuclides with short half-lives such as ${ }^{18} \mathrm{~F}$ or ${ }^{68} \mathrm{Ga}$ may be used, further augmenting the dosimetric benefits of the approach.

Ultimately, we believe that this pretargeted PET imaging methodology could be an extremely valuable and versatile clinical tool. Specifically, the success of the A33-based proof-of-concept system described here-both in terms of tumor delineation and in terms of dosimetry-has identified the system as a prime candidate for translation to the clinic for the imaging of colorectal cancer. Translation of this approach is an enticing prospect for two principal reasons. First, from a dosimetry perspective, the ${ }^{64} \mathrm{Cu} / \mathrm{A} 33$ pretargeting system would almost certainly prove a safer alternative for patients than the ${ }^{124} \mathrm{I}$-A33 PET radiotracer currently used, without undue sacrifice in image quality. Second, the targeting of A33 to normal bowel has proved a major concern in clinical trials using A33 (25). Kinetic models, however, have suggested that the halflife of the antibody in normal colon is far less than that of the antibody in the tumor. A pretargeting strategy would be a near

TABLE 2

Dosimetry Calculations for Imaging Constructs Studied

\begin{tabular}{|c|c|c|c|}
\hline Target organ* & ${ }^{64} \mathrm{Cu}$ pretargeting ${ }^{\dagger}$ & ${ }^{64} \mathrm{Cu}-\mathrm{NOTA}-\mathrm{A} 33^{\ddagger}$ & ${ }^{89} \mathrm{Zr}-\mathrm{DFO}-\mathrm{A} 33^{\S}$ \\
\hline Adrenals & 0.0068 & 0.0196 & 0.4432 \\
\hline Brain & 0.0064 & 0.0150 & 0.2065 \\
\hline Breasts & 0.0056 & 0.0138 & 0.1678 \\
\hline Gallbladder wall & 0.0074 & 0.0200 & 0.3892 \\
\hline Lower large intestine wall & 0.0449 & 0.0522 & 0.3622 \\
\hline Small intestine & 0.0089 & 0.0225 & 0.3000 \\
\hline Stomach wall & 0.0072 & 0.0239 & 0.2565 \\
\hline Upper large intestine wall & 0.0308 & 0.0392 & 0.3243 \\
\hline Heart wall & 0.0079 & 0.0292 & 0.4189 \\
\hline Kidneys & 0.0085 & 0.0503 & 0.6838 \\
\hline Liver & 0.0084 & 0.0524 & 0.7676 \\
\hline Lungs & 0.0078 & 0.0484 & 0.6108 \\
\hline Muscle & 0.0037 & 0.0148 & 0.3432 \\
\hline Ovaries & 0.0081 & 0.0184 & 0.2946 \\
\hline Pancreas & 0.0070 & 0.0191 & 0.3703 \\
\hline Red marrow & 0.0143 & 0.0832 & 0.8432 \\
\hline Osteogenic cells & 0.0230 & 0.1186 & 1.6459 \\
\hline Skin & 0.0052 & 0.0125 & 0.1830 \\
\hline Spleen & 0.0049 & 0.0324 & 0.6811 \\
\hline Testes & 0.0061 & 0.0141 & 0.1846 \\
\hline Thymus & 0.0061 & 0.0158 & 0.2670 \\
\hline Thyroid & 0.0062 & 0.0152 & 0.2559 \\
\hline Urinary bladder wall & 0.0071 & 0.0165 & 0.2232 \\
\hline Uterus & 0.0075 & 0.0176 & 0.2543 \\
\hline Total body & 0.0074 & 0.0231 & 0.3757 \\
\hline Effective dose & 0.0124 & 0.0359 & 0.4162 \\
\hline \multicolumn{4}{|c|}{$\begin{array}{l}{ }^{*} \text { Mean organ absorbed doses and effective dose are expressed in mGy/MBq and mSv/MBq, respectively. } \\
{ }^{\dagger} 100 \mu \mathrm{g} \text { of A33-TCO injected } 24 \mathrm{~h} \text { before injection of }{ }^{64} \mathrm{Cu}-\mathrm{Tz}-\mathrm{Bn}-\mathrm{NOTA} \text {. } \\
{ }^{\ddagger} 5 \mu \mathrm{g} \text { of }{ }^{64} \mathrm{Cu}-\mathrm{NOTA}-\mathrm{A} 33 \text { per injection. } \\
{ }_{5} 5 \mu \mathrm{g} \text { of }{ }^{89} \mathrm{Zr} \text {-DFO-A33 per injection. }\end{array}$} \\
\hline
\end{tabular}


ideal way to circumvent this complication, because the pretargeted radioligand could be injected after the shedding of the antibody from the normal colon but before the departure of the antibody from the cancerous tissue.

Equally important, this methodology could also have a significant impact beyond the system described here. Along these lines, the modularity of the system is an incredibly important asset: the transcyclooctene moiety can be appended to any antibody of interest, and the simplicity of the tetrazine-based radioligand facilitates the creation of a library of constructs bearing various radioisotopes. Indeed, preliminary experiments are under way in the creation of pretargeting systems for both ${ }^{89} \mathrm{Zr}$-based PET imaging and ${ }^{177} \mathrm{Lu}$ - and ${ }^{90} \mathrm{Y}$-based radiotherapy. However, the paramount value of the system plainly lies in its ability to harness the advantages of antibody-based radiopharmaceuticals while skirting their pharmacokinetic limitations. The methodology produces high tumor-to-background contrast at relatively early time points while limiting uptake in, and thus dose to, normal organs, especially compared with antibodies bearing radionuclides with long physical half-lives. Consequently, this approach may ultimately offer an effective and safer alternative to both immunoPET and immunoSPECT imaging with long-lived isotopes such as ${ }^{89} \mathrm{Zr}$, ${ }^{111} \mathrm{In}$, or ${ }^{124} \mathrm{I}$ and radioimmunotherapy with isotopes such as ${ }^{177} \mathrm{Lu},{ }^{90} \mathrm{Y}$, or ${ }^{131} \mathrm{I}$.

\section{DISCLOSURE}

The costs of publication of this article were defrayed in part by the payment of page charges. Therefore, and solely to indicate this fact, this article is hereby marked "advertisement" in accordance with 18 USC section 1734 . This work received financial support from the NIH (1F32CA1440138-01, R01EB010011, and P50CA86355) and the DOE (DE-SC0002184 and DE-SC0002456). Services provided by the MSKCC Small-Animal Imaging Core Facility were supported in part by NIH grants R24-CA83084 and P30-CA08748. No other potential conflict of interest relevant to this article was reported.

\section{ACKNOWLEDGMENTS}

We thank Dr. Steven Larson for insight and helpful conversations and the Ludwig Institute for Cancer Immunotherapy for its generous supply of huA33 antibody and SW1222 cells.

\section{REFERENCES}

1. Wu AM. Antibodies and antimatter: the resurgence of immuno-PET. J Nucl Med. 2009;50:2-5.

2. Zeglis B, Lewis JS. A practical guide to the construction of radiometallation bioconjugates for positron emission tomography. Dalton Trans. 2011;40: 6168-6195.

3. Hollander N. Bispecific antibodies for cancer therapy. Immunotherapy. 2009;1: 211-222.
4. Sharkey RM, Goldenberg DM. Advances in radioimmunotherapy in the age of molecular engineering and pretargeting. Cancer Invest. 2006;24:82-97.

5. Boerman OC, van Schaijk FG, Oyen WJG, Corstens FHM. Pretargeted radioimmunotherapy of cancer: progress step by step. J Nucl Med. 2003;44:400411.

6. Press OW, Corcoran M, Subbiah K, et al. A comparative evaluation of conventional and pretargeted radioimmunotherapy of CD20-expressing lymphoma xenografts. Blood. 2001;98:2535-2543.

7. Goldenberg DM, Chang CH, Rossi EA, McBride WJ, Sharkey RM. Pretargeted molecular imaging and radioimmunotherapy. Theranostics. 2012;2:523-540.

8. Liu G, Dou S, Liu Y, Wang Y, Rusckowski M, Hnatowich DJ. ${ }^{90}$ Y-labeled phosphorodiamidate morpholino oligomer for pretargeting radiotherapy. Bioconjug Chem. 2011;22:2539-2545.

9. Lewis MR, Wang M, Axworthy DB, et al. In vivo evaluation of pretargeted ${ }^{64} \mathrm{Cu}$ for tumor imaging and therapy. J Nucl Med. 2003;44:1284-1292.

10. Mohsin H, Jia F, Bryan JN, et al. Comparison of pretargeted and conventional CC49 radioimmunotherapy using ${ }^{149} \mathrm{Pm},{ }^{166} \mathrm{Ho}$, and ${ }^{177} \mathrm{Lu}$. Bioconjug Chem. 2011;22:2444-2452.

11. Kolb HC, Finn MG, Sharpless KB. Click chemistry: diverse chemical function from a few good reactions. Angew Chem Int Ed Engl. 2001;40:2004-2021.

12. Sletten EM, Bertozzi CR. Bioorthogonal chemistry: fishing for selectivity in a sea of functionality. Angew Chem Int Ed Engl. 2009;48:6973-6998.

13. Devaraj NK, Upadhyay R, Hatin JB, Hilderbrand SA, Weissleder R. Fast and sensitive pretargeted labeling of cancer cells through a tetrazine/trans-cyclooctene cycloaddition. Angew Chem Int Ed Engl. 2009;48:7013-7016.

14. Blackman ML, Royzen M, Fox JM. Tetrazine ligation: fast bioconjugation based on inverse electron demand Diels-Alder reactivity. J Am Chem Soc. 2008;130: 13518-13519.

15. Zeglis BM, Mohindra P, Weissmann GI, et al. Modular strategy for the construction of radiometalated antibodies for positron emission tomography based on inverse electron demand Diels-Alder click chemistry. Bioconjug Chem. 2011;22: 2048-2059.

16. Li Z, Cai H, Hassink M, et al. Tetrazine-trans-cyclooctene ligation for the rapid construction of 18-F labeled probes. Chem Commun (Camb). 2010;46:80438045.

17. Reiner T, Keliher EJ, Earley S, Marinelli B, Weissleder R. Synthesis and in vivo imaging of a ${ }^{18} \mathrm{~F}$-labeled PARP1 inhibitor using a chemically orthogonal scavenger-assisted high-performance method. Angew Chem Int Ed Engl. 2011; 50:1922-1925.

18. Rossin R, Verkerk PR, van den Bosch SM, et al. In vivo chemistry for pretargeted tumor imaging in live mice. Angew Chem Int Ed Engl. 2010;49:3375-3378.

19. Devaraj NK, Thurber GM, Keliher EJ, Marinelli B, Weissleder R. Reactive polymer enables efficient in vivo bioorthogonal chemistry. Proc Natl Acad Sci USA. 2012;109:4762-4767.

20. Carrasquillo JA, Pandi-Taskar N, O'Donoghue JA, et al. ${ }^{124}$ I-huA33 antibody PET of colorectal cancer. J Nucl Med. 2011;52:1173-1180.

21. Ackerman ME, Chalouni C, Schmidt MM, et al. A33 antigen displays persistent surface expression. Cancer Immunol Immunother. 2008;57:1017-1027.

22. Wadas TJ, Wong EH, Weisman GR, Anderson CJ. Coordinating radiometals of copper, gallium, indium, yttrium, and zirconium for PET and SPECT imaging of disease. Chem Rev. 2010;110:2858-2902.

23. Heneweer C, Holland JP, Divilov V, Carlin S, Lewis JS. Magnitude of enhanced permeability and retention effect in tumors with different phenotypes: $\mathrm{Zr}$-89albumin as a model system. J Nucl Med. 2011;52:625-633.

24. Stabin MG, Sarks RB, Crowe E. OLINDA/EXM: the second-generation personal computer software for internal dose assessment in nuclear medicine. J Nucl Med. 2005;46:1023-1027.

25. Welt S, Scott AM, Divgi CR, et al. Phase I/II study of iodine 125-labeled monoclonal antibody A33 in patients with advanced colon cancer. J Clin Oncol. 1996;14:1787-1797. 